\section{Tratamiento odontológico multidisciplinario de un paciente complejo con fisura labiopalatina bilateral}

\section{Multidisciplinary dental treatment of a complex patient with bilateral cleft lip and palate}

\begin{abstract}
Resumen
La fisura labiopalatina es la malformación congénita más común entre las anomalías de cabeza y cuello. Impacta directamente en la calidad de vida del paciente y su familia por lo que el manejo multidisciplinario y oportuno de sus secuelas resulta fundamental para el crecimiento y desarrollo del paciente. El objetivo de este caso clínico fue mostrar el manejo odontológico integral de una paciente con fisura labiopalatina bilateral, sexo femenino, 14 ańos de edad, que acudió junto a su madre al Instituto de Malformaciones Craneofaciales de la Universidad de Chile porque deseaban una solución a su estética facial alterada y corrección de la malposición dentaria. Mediante el manejo multidisciplinario se buscó alcanzar un adecuado funcionamiento del sistema estomatognático y corregir la estética para lo cual fue sometida a un tratamiento ortodóncico, quirúrgico y rehabilitador. Tras ello se obtuvieron resultados positivos en relación a una oclusión funcional y una satisfactoria estética dental y facial. En conclusión, el manejo multidisciplinario plantea un desafío para el odontólogo desde el momento en que se desea obtener resultados favorables brindando un enfoque más amplio de las alternativas de tratamiento para los pacientes, lo cual no implica optar por el tratamiento ideal, sino más bien por la mejor alternativa tanto funcional como estética para que el paciente pueda escoger lo adecuado, según sus posibilidades y expectativas.
\end{abstract}

Palabras clave: Cirugía ortognática; Ortodoncia; Paladar hendido.

\section{Caso Clínico}

Noemí Leiva Villagra ${ }^{1, a, b}$, Loreto Saavedra Layera $^{1, a, c}$, Roberto Pantoja Parada ${ }^{1, d}$

${ }^{1}$ Facultad de Odontología. Universidad de Chile. Santiago, Chile.

a Cirujana Dentista

${ }^{\mathrm{b}}$ Especialista en Ortodoncia y Ortopedia Maxilofacial

c Diplomada en Ortodoncia Preventiva e Interceptiva

dCirujano Maxilofacial

Correspondencia:

Noemí Leiva Villagra

Correo electrónico: leivanoemi@yahoo.com

General Holley 2381, oficina 606, Providencia, Santiago, Chile.

\section{Coautores:}

Loreto Saavedra Layera

loreslayera@gmail.com

Roberto Pantoja Parada

profepantoja@gmail.com

Conflicto de intereses: Los autores declaran no tener conflictos de interés.

Fuente de financiamiento: Autofinanciado.

Fecha de recepción: 19/07/17

Fecha de aceptación: 02/09/17

\begin{abstract}
The cleft lip and palate is the most common congenital malformation between head and neck anomalies. It has an impact on the quality of life of the patient and his family, therefore a multidisciplinary and timely management of its sequels is fundamental for his development. The purpose of this clinical case was to show the integral dental management of a patient diagnosed with bilateral cleft lip and palate, female, 14 years old, who went with her mother to the Institute of Craniofacial Malformations of the University of Chile because they wanted a solution for her altered facial aesthetics and correction of dental malposition. Through the multidisciplinary management it was expected to achieve an adequate functioning of the stomatognathic system and to correct the esthetics for which it was subjected to an orthodontic, surgical and rehabilitating treatment. After this, positive results were obtained in terms of a functional occlusion and a satisfactory dental and facial aesthetic. In conclusion, multidisciplinary management presents a challenge to a dentist from the moment in which he desires to obtain satisfactory results by offering a wider approach of the treatment alternatives for his patients. This does not imply to choose an ideal treatment but rather the best functional and aesthetic alternative; hence the patient can choose the right one, according to their means and expectations.
\end{abstract}

Keywords: Cleft palate; Orthodontics; Orthognathic surgery.

(C) Los autores. Este artículo es publicado por la revista Odontología Sanmarquina de la Facultad de Odontología, Universidad Nacional Mayor de San Marcos. Este es un artículo de acceso abierto, distribuido bajo los términos de la licencia Creative Commons Atribucion - No Comercia_Compartir Igual 4.0 Internacional. (http://creativecommons.org/licenses/by-nc-sa/4.0/) que permite el uso no comercial, distribución y reproducción en cualquier medio, siempre que la obra original sea debidamente citada. 


\section{Introducción}

La fisura labiopalatina corresponde a una inadecuada fusión durante el desarrollo embrionario de los procesos que dan origen a las cavidades oral y nasal. Esto genera una alteración en la anatomía del labio superior y/o del paladar dando como consecuencia una serie de alteraciones a nivel funcional, psicológico y social ${ }^{1}$.

Su incidencia a nivel mundial es 0,83 por cada mil recién nacidos vivos $(\mathrm{RNV})^{2}$, en Chile su mayor incidencia se estimó en 1,78 por cada mil RNV, transformándose en el trastorno congénito maxilofacial más frecuente ${ }^{3}$. La presentación de esta anomalía puede afectar al paciente unilateral o bilateralmente. Nótese que del total de pacientes afectados con fisura labiopalatina en Chile, 25\% corresponden a fisurados bilaterales ${ }^{4}$.

Su etiología no se conoce a cabalidad, sin embargo, se asocia como multifactorial, incidiendo en su aparición tanto factores ambientales (anticonvulsivantes, vitamina $A$, corticoides, infecciones virales durante el primer trimestre de embarazo) presentes en alrededor de un 20 $25 \%$ de los pacientes; como genéticos, observados en un $20-25 \%$ de los casos. En el resto no se ha encontrado causa precisa ${ }^{3,5}$.

Los pacientes afectados por fisuras de labio y/o paladar frecuentemente se caracterizan por presentar alteradas la anatomía y las funciones que el sistema estomatognático desarrolla, además de presentar un compromiso psicológico y social en distinto grado. Por tal motivo, el plan de tratamiento requiere un manejo multidisciplinario para el cual se deben considerar cirugía maxilofacial, ortodoncia, rehabilitación oral, otorrinolaringología, cirugía plástica, fonoaudiología y el manejo psicológico tanto del paciente como de la familia ${ }^{1}$. La evaluación se debe iniciar en el periodo de recién nacido. Las cirugías primarias que corresponden al cierre de labio y paladar, tienen por finalidad cerrar la fisura, separando la cavidad oral de la nasal y junto a ello, alcanzar un resultado estético y funcional adecuado ${ }^{6,7}$. Posteriormente, pueden ser necesarias cirugías secundarias para la corrección de los defectos que se manifiesten durante el desarrollo del paciente, tales como cierre de fístulas de paladar, faringoplastía, retoques de labio y nariz, injertos óseos, rinoplastias definitivas y osteotomías maxilares con o sin distracción ósea ${ }^{6}$.

A continuación se presenta un caso clínico de una paciente con fisura labiopalatina bilateral resuelto por un equipo multidisciplinario que involucró ortodoncia, cirugía maxilofacial y rehabilitación oral, además de medidas preventivas odontológicas muy bien cumplidas por la paciente y su cuidadora, la madre.

\section{Reporte del caso}

Paciente sexo femenino, 14 años de edad. Consulta por evaluación y posibilidad de tratamiento rehabilitador integral. Diagnosticada con labio leporino y reborde bilateral operado. Se realizó cirugía de labio en dos oportunidades y se sometió a cirugía para alargamiento de columela y gingivoperiosteoplastía derecha. Entre sus antecedentes al momento de nacer se encuentran los diagnósticos de tortícolis, displasia de cadera, hemidistonía derecha, alteración en el coeficiente intelectual marcado. Al examen extraoral se observa labio superior corto con secuela cicatrizal, labio inferior evertido, labio superior fino, leve asimetría facial (Figura 1).

El examen clínico intraoral reveló compresión maxilar, ausencia de piezas dentarias 1.2 y 2.2 , comprometiendo en gran medida la estética facial, desviación de línea media dentaria inferior hacia la izquierda, mordida invertida y mordida bis a bis a nivel de pieza 1.4, mordida cruzada izquierda a nivel molar y en relación a piezas dentales 1.5 y 1.6; mesioclusión molar bilateral (Figura 2).

En la radiografía panorámica se observa dentición mixta segunda fase, ausencia congénita de los incisivos laterales superiores bilaterales (Figura 3). El análisis cefalométrico reveló una paciente con clase III esqueletal por retrusión del maxilar superior y protrusión de la mandíbula (Figura 4).

Los objetivos del tratamiento fueron alcanzar una oclusión funcional, mejorar la estética facial, dental y junto a ello, su calidad de vida. Se inició el tratamiento ortodóncico con aparatos fijos en ambas arcadas con el objetivo de alinear y nivelar ambas arcadas dentarias para posteriormente realizar la corrección de la alteración esqueletal (Figuras 4 y 5 ).

Durante la adolescencia fue sometida a una cirugía ortognática de retrusión mandibular de acuerdo al análisis para luego continuar con el tratamiento ortodóncico (Figura 6). De esta manera, se alcanzó una adecuada relación de las arcadas y una oclusión estable y funcional (Figuras 7 y 8 ).

Una vez concluido el tratamiento ortodóncico fue derivada a rehabilitación oral donde se confeccionó una prótesis parcial removible a modo de contención y para devolver la estética en relación a los espacios desdentados correspondientes a las piezas dentales 1.2 y 2.2 (Figura 9). A través de este aparato se logró mantener el tratamiento efectuado y mejorar de un modo importante la sonrisa.

\section{Discusión}

La fisura labiopalatina bilateral afecta al labio y paladar primario de ambos lados del maxilar. Involucra un compromiso aún mayor de los labios y proceso alveolar, el cual tiene una extensa deformidad, creando severos problemas al visualizar la arcada y funcional.

Estos pacientes presentan múltiples secuelas, y el tratamiento exitoso implica un equipo multidisciplinario pero también una evaluación periódica del progreso, pudiendo ser necesarias modificaciones del plan original ya sea porque las necesidades y prioridades del paciente cambian o porque las etapas varían ${ }^{8}$.

Los problemas más frecuentemente encontrados en pacientes afectados con fisura labiopalatina son: agenesia de incisivos laterales, falta de desarrollo maxilar en algu- 

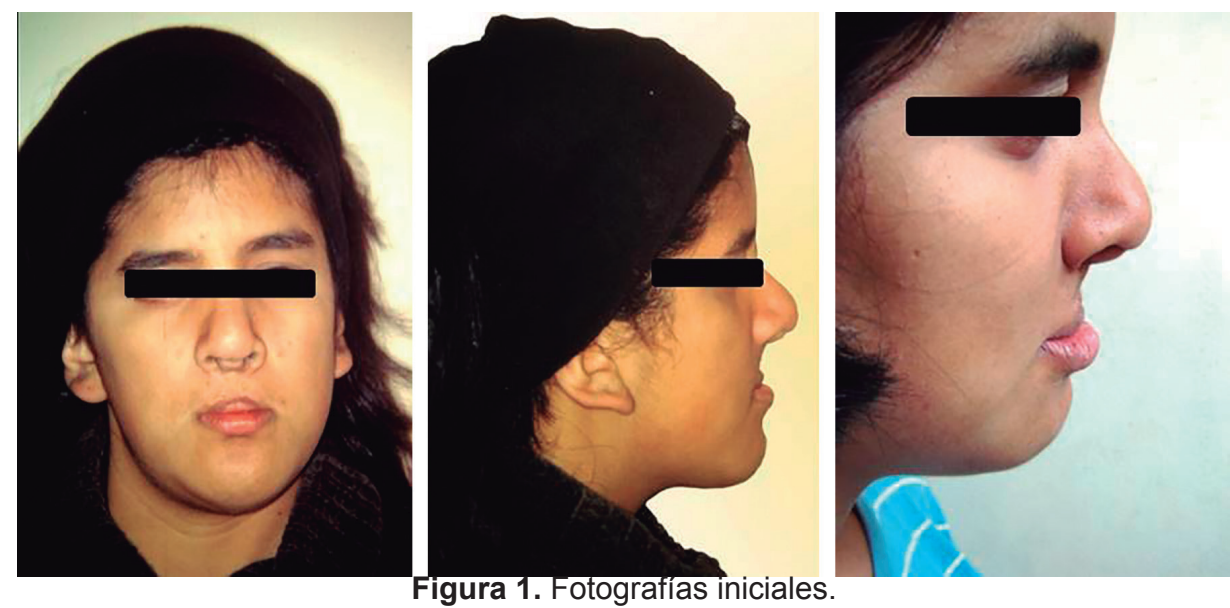

Figura 1. Fotografias iniciales.
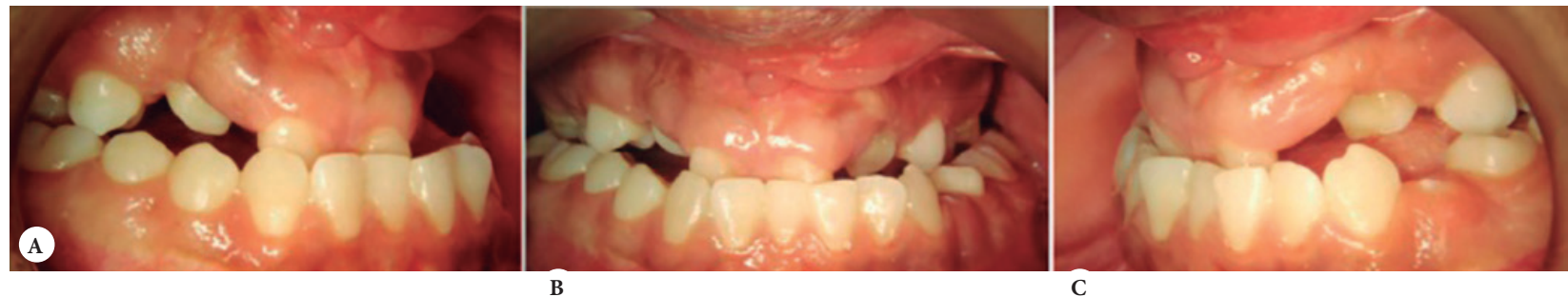

Figura 2. Fotografías intraorales. A: vista lateral derecha. B: vista frontal. C: vista lateral izquierda.

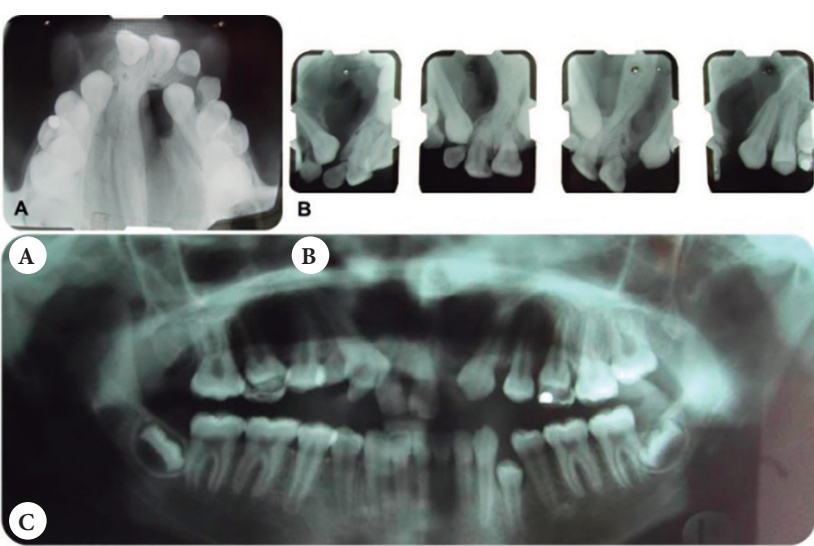

Figura 3. Radiografías previas al tratamiento. A: vista oclusal. B: radiografías periapicales del área de la fisura bilateral. C: radiografía panorámica.
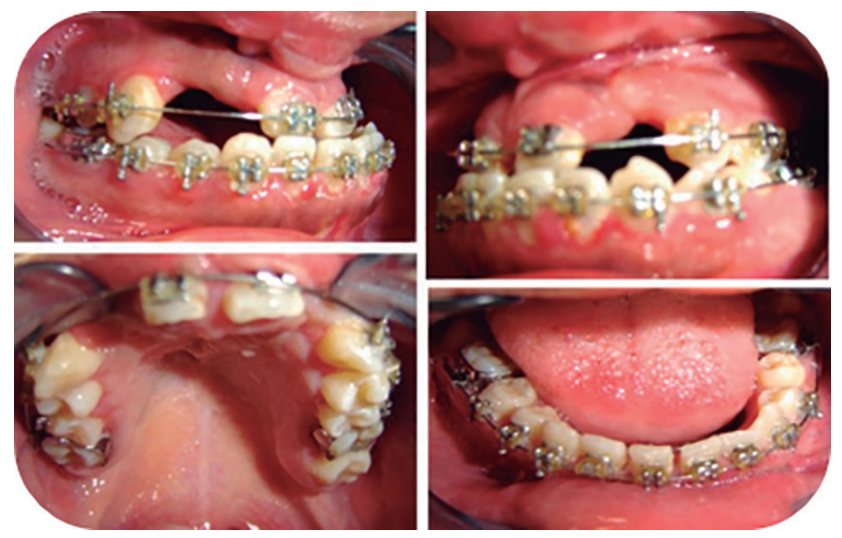

Figura 5. Fotografías intraorales durante el tratamiento ortodóncico.
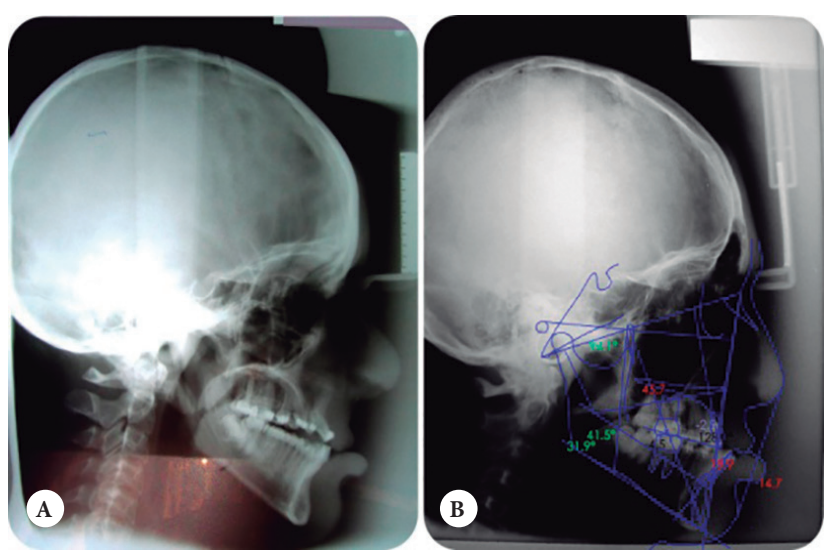

Figura 4. A: telerradiografía lateral previa al tratamiento B: trazado cefalométrico de Ricketts.
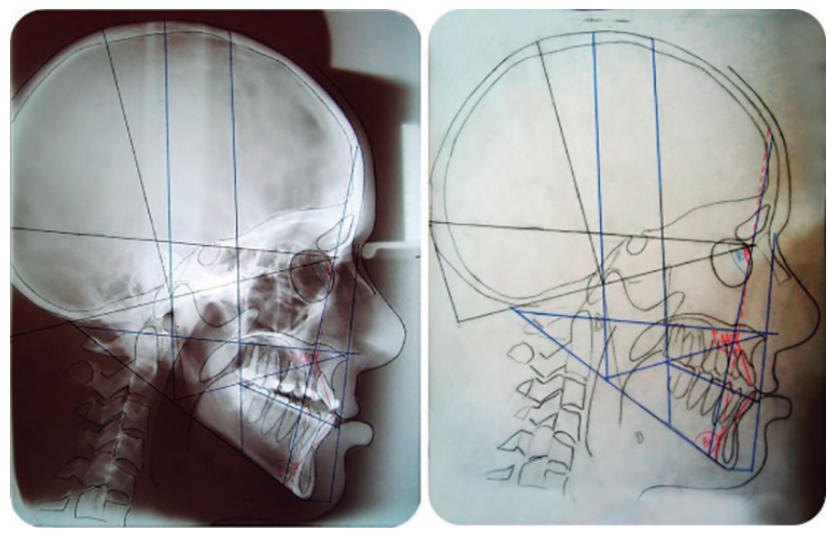

Figura 6. Telerradiografía lateral previo y post tratamiento quirúrgico. 

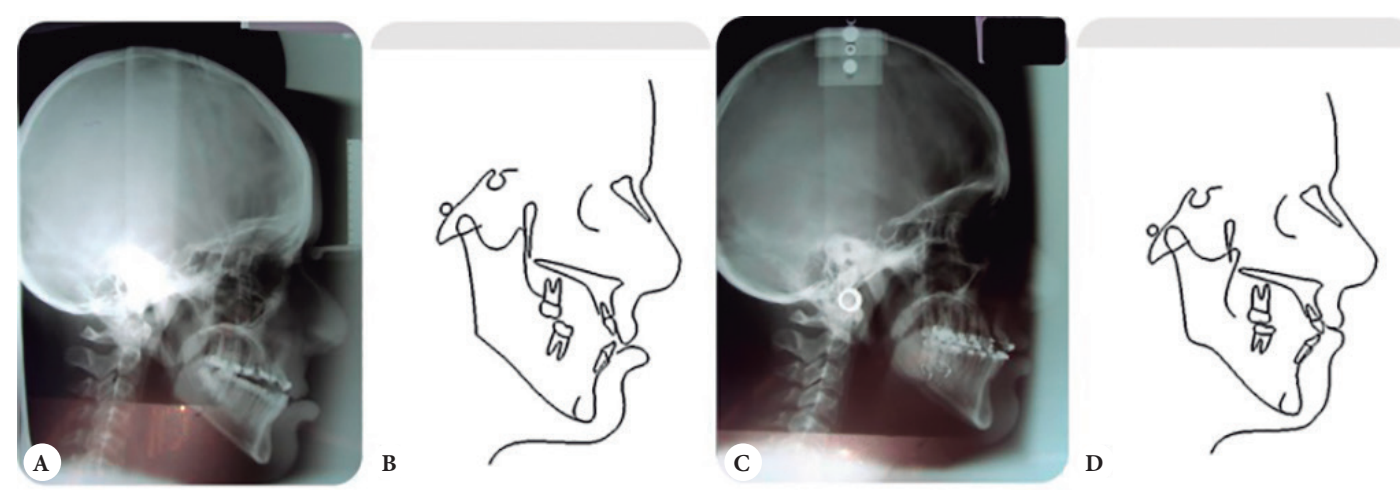

Figura 7. A: telerradiografía lateral previa a cirugía ortognática de retrusión mandibular. B: trazado cefalométrico anterior a procedimiento quirúrgico. C: telerradiografía posterior a tratamiento quirúrgico. D: trazado cefalométrico posterior a cirugía ortognática. Nótese la mejoría en el perfil

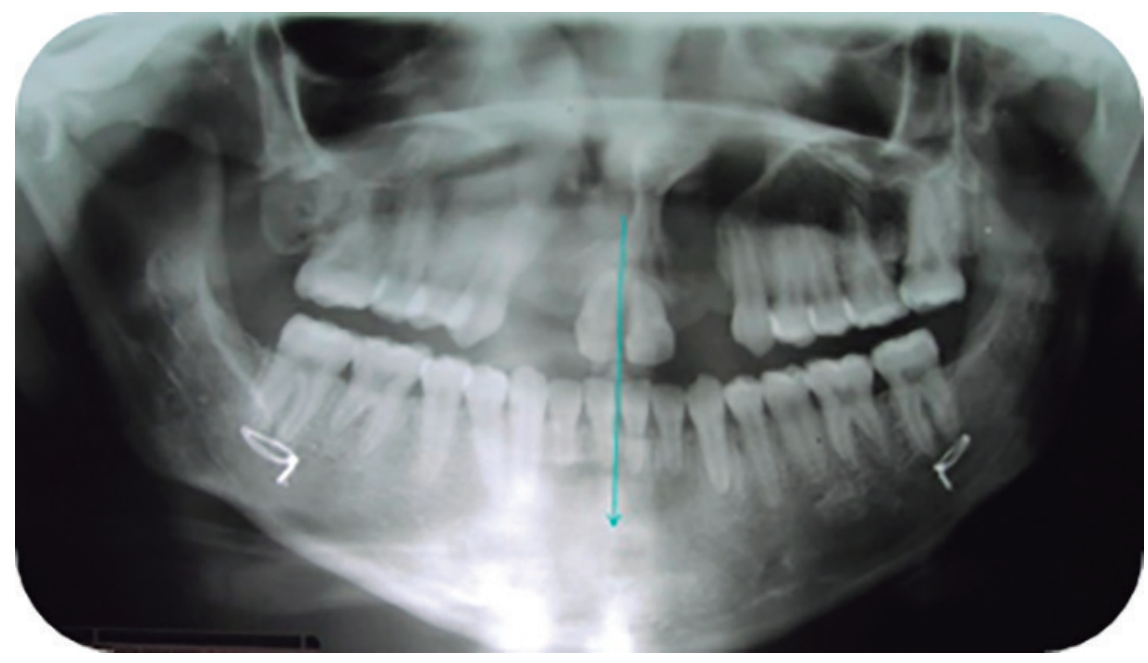

Figura 8. Radiografía panorámica final.
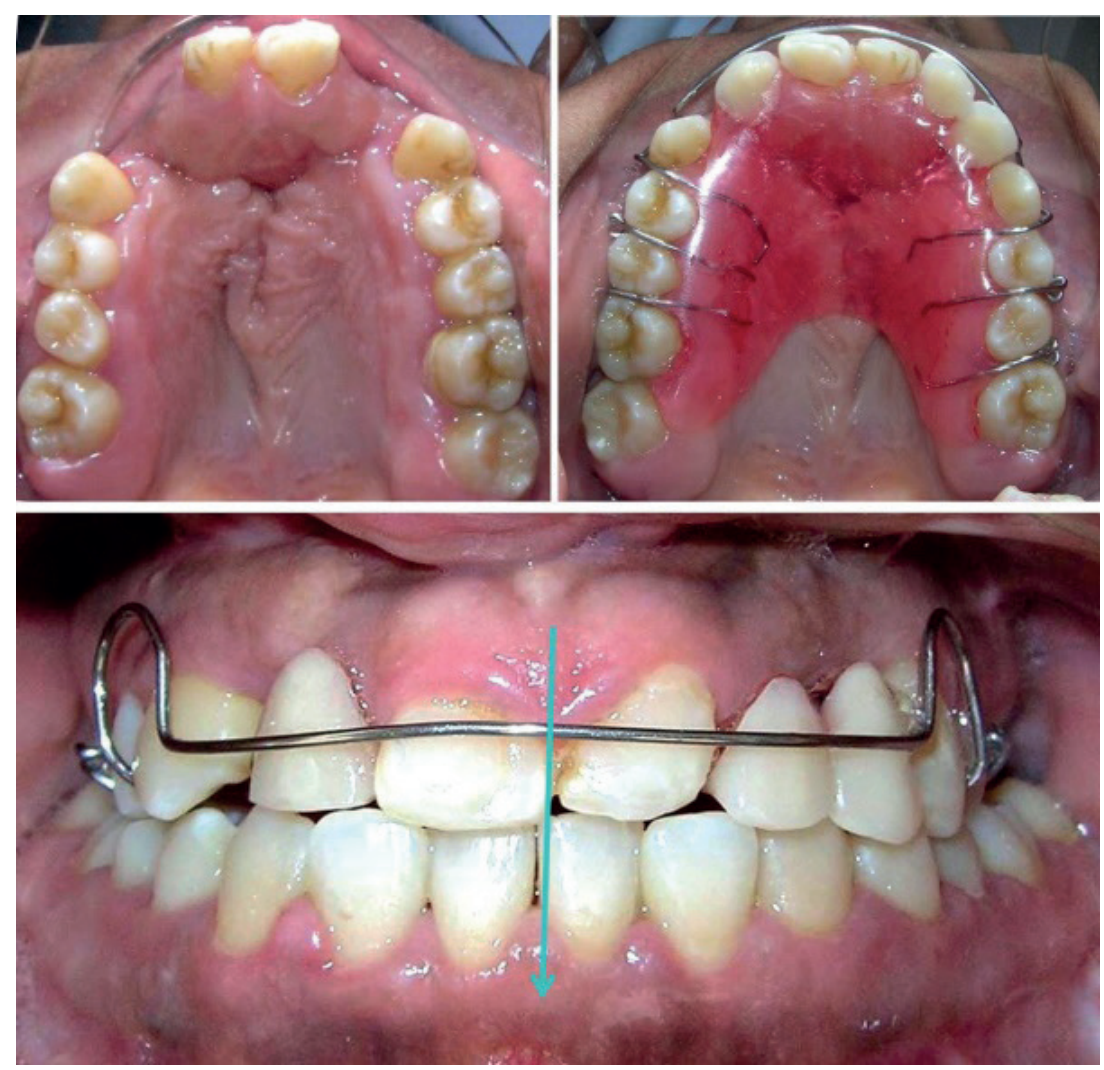

Figura 9. Fotografías intraorales finales del tratamiento. 
no de los sentidos del espacio, discrepancia entre el tamaño dentario y de los maxilares y dientes impactados?

Para determinar el tratamiento más adecuado en cada caso, es necesario evaluar cuidadosamente las características clínicas y radiológicas para determinar si el paciente necesitará solo tratamiento ortodóncico o complementarlo con cirugía ortognática ${ }^{9}$.

Diversos estudios señalan que las anomalías dentales son más frecuentes en pacientes con fisura labiopalatina que en la población en general ${ }^{9,10}$. La agenesia de los incisivos laterales superiores permanentes es un problema común en estos pacientes y el manejo de esta situación implica decidir si cerrar el espacio, posicionando en su lugar el canino permanente, o crearlo para reemplazar posteriormente el diente ausente mediante una rehabilitación, lo que debe escogerse sobre una base individual $^{8,9}$. Rioux y cols. ${ }^{9}$ señalan que generalmente estos pacientes presentan una fuerte tendencia a la Clase III debido a un deficiente crecimiento maxilar tanto sagital como vertical, combinado con una autorrotación de la mandíbula. Ante este escenario, describe que cerrar un espacio desdentado en el maxilar empeorará una Clase III y, por el contrario, abrir un espacio proporcionaría un resultado estético satisfactorio con una sonrisa más amplia y mayor soporte para el labio superior. En el caso desarrollado, la paciente tenía ausencia congénita de las piezas dentales adyacentes a la fisura, los incisivos laterales superiores. Esta situación hizo más compleja la planificación del tratamiento, ya que se debía alcanzar un resultado oclusal que fuera estético pero al mismo tiempo, funcional y estable. Finalmente, se decidió llevar a una correcta posición los caninos superiores para posteriormente rehabilitar el espacio desdentado correspondiente a los incisivos laterales permanentes ausentes.

La compresión maxilar y mordida cruzada son hallazgos comunes en pacientes con fisura labiopalatina ${ }^{8}$. Entre las opciones para corregir estos problemas podemos mencionar la expansión maxilar, expansión ortodóncica asistida quirúrgicamente, distracción transpalatal y expansión quirúrgica. Teniendo en consideración las bases óseas y el grado de entrecruzamiento, se decidió corregir ortodóncicamente la mordida bis a bis y cruzada posterior, mientras que la corrección de la marcada alteración sagital se realizó quirúrgicamente.

Las formas clínicas de las secuelas que pueden presentar estos pacientes, son variadas y difieren según el tratamiento y seguimiento recibidos ${ }^{11}$. Los pacientes afectados con fisura labiopalatina suelen presentar una clase lll esqueletal debido a un deficiente desarrollo del maxilar que puede estar combinado con un componente mandibular como se observa en el caso expuesto. En un estudio llevado a cabo por Good y cols. ${ }^{12}$ reportaron la incidencia de la hipoplasia maxilar en un $21 \%$ de los pacientes con fisura labiopalatina. Aquellos con fisura labiopalatina no sindrómica, la incidencia de trastornos del crecimiento mandibular se presenta similar a la de la población normal ${ }^{13}$. Para la corrección de este tipo de defectos esqueletales, la cirugía ortognática es una alternativa terapéutica. Se ha observado que el porcen- taje de pacientes con fisura labiopalatinas que requieren cirugía ortognática para corregir las maloclusiones clase III es mayor que en la población general $(25 \%){ }^{9}$. Taib y cols. ${ }^{14}$ seńalan que la cirugía ortognática para corregir la alteración del crecimiento facial está indicada en hasta un $80 \%$ de los pacientes. Ésta puede abarcar una combinación de cirugía maxilar, mandibular y de mentón para corregir los defectos esqueléticos y oclusales en el paciente afectado con fisura labiopalatina. En el caso presentado, fue necesario planificar una cirugía ortognática de retrusión mandibular posterior a la preparación ortodóncica. Ésta fue llevada a cabo una vez finalizado el crecimiento de la paciente y consistió en una cirugía de retroceso mandibular con osteotomía sagital de la rama mandibular bilateral para corregir la discrepancia anteroposterior. El reposicionamiento de la mandíbula en los tres planos del espacio ayudó a restablecer el equilibrio dental, facial y proporcionar estabilidad.

Luego del tratamiento ortodóncico quirúrgico es común que los pacientes con fisura labiopalatina requieran una rehabilitación protésica planificada en conjunto: ortodoncista y rehabilitador oral. Existen diversas alternativas para pacientes con piezas dentales ausentes en el sitio de la fisura que van a depender de la ubicación del área, la presencia de suficiente hueso alveolar y tejido gingival, y de la estabilidad de los segmentos maxilares. Entre las opciones podemos mencionar la rehabilitación protésica removible, fija o combinada ${ }^{8}$. La elección y ejecución debe ser planificada considerando los aspectos anteriormente mencionados pero también teniendo en consideración alcanzar una rehabilitación integral que comprenda los componentes físicos, psíquicos y emocionales del paciente.

En el caso clínico desarrollado, se optó por la confección de una prótesis parcial removible superior que reemplazara las piezas dentales ausentes, normalizara la oclusión y a su vez actuara como contención para el tratamiento ortodóncico llevado a cabo. Se puede señalar que la rehabilitación oral mediante prótesis parcial removible correctamente diseñada y confeccionada entrega a los pacientes una alternativa de tratamiento y una solución eficaz tanto funcional como estética.

Es importante mencionar que el estado periodontal debe ser evaluado previamente, ya que es frecuente pesquisar como secuela en los pacientes con fisura labiopalatina un defecto en la cresta alveolar de los dientes vecinos a la fisura, con marcada recesión gingival y que es difícil de corregir ${ }^{15}$.

El seguimiento de estos pacientes debe realizarse durante varios años, hasta el completo desarrollo y maduración del esqueleto. Durante todo el proceso deben ser evaluados por los diferentes profesionales quienes ejecutarán intervenciones oportunamente ${ }^{15}$. En este caso, tras el seguimiento se pudo observar que la prótesis parcial removible permaneció en adecuadas condiciones, no se observaron complicaciones.

En conclusión, este reporte clínico permite tener presente que la ortodoncia y la cirugía ortognática deben 
trabajar en conjunto para estos casos donde la clase III esqueletal definió la terapia quirúrgica mandibular y que el manejo multidisciplinario plantea un tremendo desafío al odontólogo desde el momento en que se desean obtener resultados favorables brindando un enfoque más amplio de las alternativas de tratamiento para los pacientes, lo cual implica no solo optar por el tratamiento ideal, sino más bien la mejor alternativa tanto funcional como estética y escoger la más adecuada.

\section{Referencias bibliográficas}

1. Cordero E, Correa S, Pantoja R. Prevalence of Patients With Cleft Lip and Palate who were Treated at the San Borja Arriarán Clinical Hospital in Santiago Chile, within the AUGE Healthcare Plan Int J Odontostomat. 2015;9(3):469-73. Doi: 10.4067/S0718$381 X 2015000300018$.

2. Cooper ME, Ratay JS, Marazita ML. Asian oral-facial cleft birth prevalence. Cleft Palate Craniofac J. 2006;43(5):580-9. Doi: 10.1597 / 05-167

3. Nazer J, Constanza M, Cifuentes L. Evolution of prevalence rates of orofacial clefts in a maternity of a Chilean clinical hospital. Rev Med Chile. 2010;138:567-72. Doi: S0034-9887201000050000

4. Sepúlveda G, Palomino H, Cortés J. Prevalencia de fisura labiopalatina e indicadores de riesgo: Estudio de la población atendida en el Hospital Clínico Félix Bulnes de Santiago de Chile. Rev Esp Cirug Oral y Maxilofac. 2008;30(1):17-25.

5. Ford A. Tratamiento actual de las fisuras labio-palatinas. Rev Med Clin Condes. 2004;15(1):3-11.

6. Ford A, Tastets M, Caceres A. Treatment of patients with cleft lip and palate. Rev Med Clin Condes. 2010;21(1):16-25. Doi: 10.1016/S0716-8640(10) 70501-0

7. Leiva N, Nieto E, Véliz S, Valle M. Prosthetic rehabilitation in patients with cleft lip and buconasal fistula. Rev Clin Periodoncia Implantol Rehabil Oral. 2016; 9(3):222-5. Doi: 10.1016/j.piro.2015.09.010.
8. Germec-Cakan D, Canter HI, Cakan U, Demir B. Interdisciplinary treatment of a patient with bilateral cleft lip and palate and congenitally missing and transposed teeth. Am J Orthod Dentofacial Orthop. 2014;145:38192. Doi: 10.1016/j.ajodo.2013.06.021.

9. Rioux E, Decker A, Deffrennes D. Therapeutic thoughts on the treatment of sequellae of labial-alveolar-palatal clefts in adult patients - part 2. Int Orthod. 2012;10(4):404-21. Doi: 10.1016 / j.ortho.2012. 09.009

10. Ranta R. A review of tooth formation in children with cleft lip/palate. Am J Orthod Dentofacial Orthop. 1986;90:11-8.

11. Rioux E, Decker A, Deffrennes D. Therapeutic thoughts on the treatment of sequellae of labial-alveolar-palatal clefts in adult patients - part 1. Int Orthod. 2012;10:24160. Doi: 10.1016 / j.ortho.2012.07.001.

12. Good P, Mulliken J, Padwa B. Frequency of Le Fort I osteotomy after repaired cleft lip and palate or cleft palate. Cleft Palate Craniofac J. 2007;44:396.

13. James J, Costello B, Ruiz R. Management of Cleft Lip and Palate and Cleft Orthognathic Considerations. Oral Maxillofac Surg Clin. 2014;26(4):565-572. Doi: 10.1597/06-075.1

14. Taib B, Taib A, Swift A, Van Eeden S. Cleft Lip and Palate: Diagnosis and Management. Br J Hosp Med. 2015;76(10):584-91. Doi: 10.12968 / hmed.2015.76. 10.584

15. Leiva N, Nieto E, Vergara C, Salas M. Prosthesis and orthodontics. Bilateral fissure repair: A clinical case. Rev Clin Periodoncia Implantol Rehabil Oral. 2017;10(1):14-6. Doi: 10.1016/j.piro.2015.06.006. 